\title{
Cloud microphysical characterization during AVIRIS-NG campaign
}

\author{
Bipasha Paul Shukla ${ }^{1 *}$, Jinya John ${ }^{1,2}$ and Sambit Kumar Panda ${ }^{1}$ \\ ${ }^{1}$ Atmospheric Sciences Division, Atmosphere and Oceanic Sciences Group, Space Applications Centre, Indian Space Research Organisation, \\ Ahmedabad 380 015, India \\ ${ }^{2}$ Department of Physics, Electronics and Space Sciences, Gujarat University, Navrangpura, Ahmedabad 380 009, India
}

\begin{abstract}
Airborne Visible/Infrared Imaging Spectrometer-Next Generation (AVIRIS-NG) air campaign has provided a unique opportunity to characterize the properties of tropical clouds at microscale. A novel approach based on spectral matching technique has been used to derive the cloud microphysical parameters (CMPs) such as optical thickness and effective radius over campaign sites of Kurnool (Andhra Pradesh) and Chilika (Odisha) region in India. It is found that the derived CMPs correspond to medium opacity and effective radius ranging from 4 to $18 \mu \mathrm{m}$. The hyperspectral bands coupled with high spatial resolution of the observations make it possible to identify pockets populated densely with large particles within a cloud. This has great applications for picking up fast developing convective cloud cells. More insight with different cloud type observations is anticipated with AVIRISNG phase-2 campaign.
\end{abstract}

Keywords: Cloud microphysical parameters, hyperspectral imaging, remote sensing, spectral matching.

\section{Introduction}

Hyperspectral remote sensing has proven to be one of the most advanced techniques with immense potential for applications in various fields of Earth observations and climate studies. It was Goetz and co-workers who introduced hyperspectral imaging spectroscopy for remote sensing applications ${ }^{1}$. With the utilization of contiguous registered spectral bands in the observation platforms, the hyperspectral data provide high spectral/spatial resolution corresponding to each pixel in the image/data, which provides a great deal of information about the target. They allow for the correct detection and identification of materials/elements which otherwise are unexplored in multispectral observations. The hyperspectral remote sensing covers all spectral domains [i.e. VIS (visible), NIR (near infrared), SWIR (shortwave infrared), MWIR (mid wave infrared) and LWIR (long wave infrared)], all spatial domains and platforms (ground, air and space), and all targets (solid, liquid and gas) ${ }^{2}$. Several remote sensing studies have been carried out for the classification of

\footnotetext{
*For correspondence. (e-mail: bipasha@sac.isro.gov.in)
}

urban surface materials, detection of hydrocarbons, identification of vegetation and water resources-based investigations to mention a few ${ }^{3-6}$, by exploiting hyperspectral data. A less explored, but useful application of hyperspectral data is regarding cloud characterization. An accurate representation of the cloud radiative and physical properties is important for climate modelling and weather predictions. These in turn are described with the help of cloud macrophysical (viz. cloud top, cloud fraction, cloud height, etc.) and microphysical properties (viz. cloud effective radius, cloud optical thickness, etc.). Therefore, it is crucial to understand the cloud properties to have deeper insights into several atmospheric phenomena as well as for better predictions ${ }^{7-9}$. However, observations and retrieval of cloud microphysical parameters (CMPs) over the Indian region are scarce ${ }^{9}$. The hyperspectral imaging and spectroscopic techniques provide unique tools for their retrieval from space, air and ground-based studies ${ }^{10}$, with a combined usage of radiative transfer simulations and spectral matching techniques using the hyperspectral data. On introducing hyperspectral measurements, information content of the derived parameter increases and the uncertainty in the retrieval reduces ${ }^{11}$.

In the present study, we explore the hyperspectral information available from Airborne Visible/Infrared Imaging Spectrometer-Next Generation (AVIRIS-NG) to understand and retrieve CMPs. We focus on two CMPs, viz. cloud optical thickness (COT) and cloud effective radius (CER).

\section{Data used and area of study}

AVIRIS-NG is a sensor developed and operated by NASA/JPL team, which provides data in 425 contiguous bands from 400 to $2500 \mathrm{~nm}$ with a $5 \mathrm{~nm}$ spectral sampling; a spatial sampling of $0.3-4 \mathrm{~m}$ has been used for the present work. Data acquisition has been in the form of a hyperspectral cube, where the $x$-, $y$ - and $z$-axis represent the scan, pixel and wavelength respectively ${ }^{12}$. AVIRISNG held its Indian campaign from 16 December 2015 to 6 March 2016. The flights were carried over by ISRO aircraft Super King Air (SKA) B200 (manufactured by Hawker Beechraft, Kansas, United States), having a 
maximum speed of $536 \mathrm{~km} / \mathrm{h}$. The aircraft has a cut-out at the centre of the cabin covered with an optical flat of $68.5 \mathrm{~cm} \times 61 \mathrm{~cm}$ for sensor operations in VIS and NIR range. Data were acquired for 51 of 57 planned Indian sites with more than 280 flight lines. Among these sites, five have been identified to be cloudy and two of them, i.e. Chilika lagoon, Odisha (25 December 2015) and Kurnool, Andhra Pradesh (26 January 2016) have been considered for this study (Figure 1). The main objective of data acquisition spans atmosphere and ocean, coastal zone, Asian soils, Asian forests, hydrocarbon alteration, mineralogy, agriculture, urban and calibration/validation studies. Several studies have been carried out which exploit the capability of AVIRIS-NG in atmospheric and oceanic applications ${ }^{13,14}$. They include estimating anthropogenic methane emission $^{15}$, atmospheric correction ${ }^{16}$, etc.

\section{Methodology}

In general, characterization and identification of target properties in hyperspectral imaging spectroscopy is done

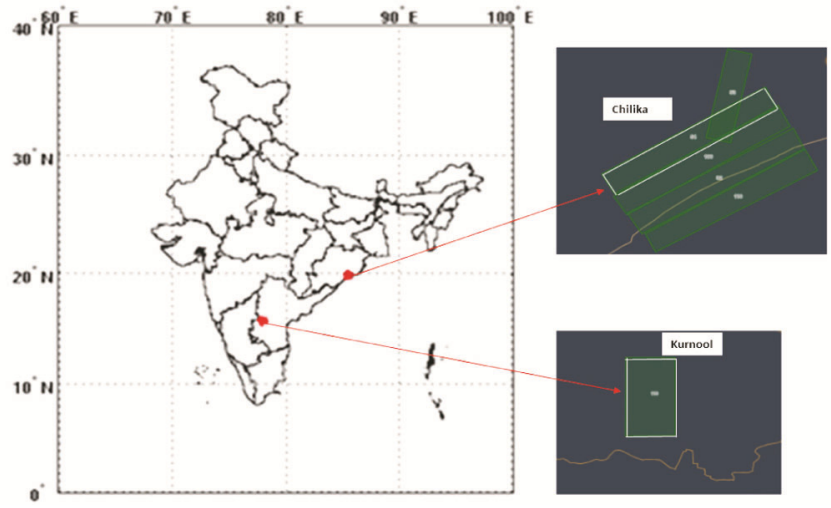

Figure 1. Study area showing Chilika lagoon $\left(19.84^{\circ} \mathrm{N}, 85.47^{\circ} \mathrm{E}\right)$ and Kurnool $\left(15.82^{\circ} \mathrm{N}, 78.03^{\circ} \mathrm{E}\right)$ regions. The flight paths are marked for the two sites.

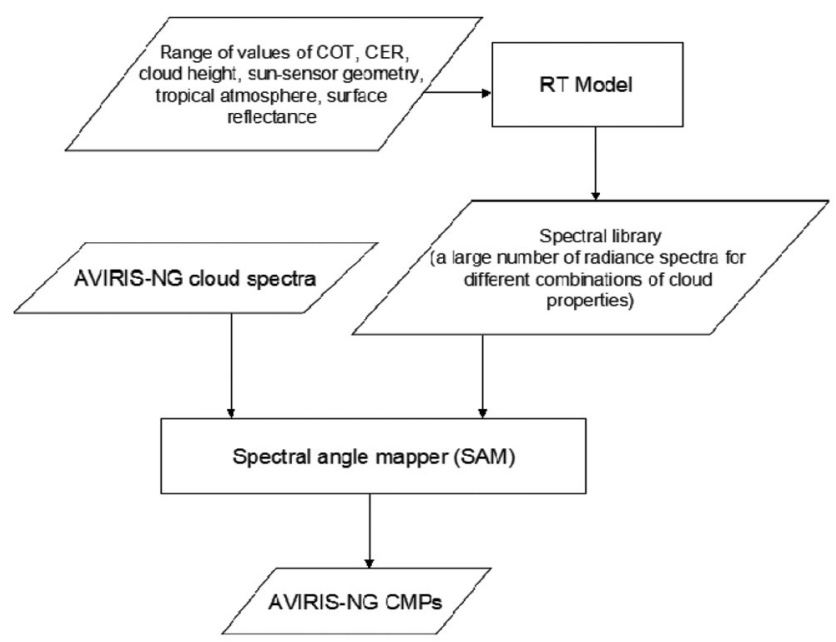

Figure 2. Flow chart of cloud microphysical parameters estimation process. by matching the observed spectra to known reference spectra. Spectral libraries which are a collection of thousands of reflectance spectra contain signatures of minerals, rocks, vegetation, man-made materials, etc. ${ }^{17,18}$. In this study, we adopt a similar procedure for CMP retrieval. However, as of now, there is no library consisting of spectra of clouds. Therefore, we generate a set of cloud spectra using a radiative transfer (RT) model to compare the observed and generated spectra in order to estimate the cloud parameters. The comparison is done using spectral classification methods; Figure 2 shows a flow chart.

\section{RT model}

Here we have used the libRadtran (library for radiative transfer) model. It uses the uvspec radiative transfer tool which computes the radiation field for a wide range of atmospheric conditions spanning the full solar and thermal spectrum from $120 \mathrm{~nm}$ to $100 \mu \mathrm{m}$ (ref. 19). The model transforms atmospheric profiles of trace gases, aerosols, water and ice clouds into optical properties such as single scatter albedo, phase function, etc. These are the inputs along with the boundary conditions (solar spectrum of atmosphere and reflecting surface at the bottom) to the radiative transfer solver. Another important feature is that it uses about ten different radiative transfer solvers which take into account the polarization effect of radiation fields and others which handle spherical, pseudospherical and plane-parallel geometry. It provides several other utilities to calculate sun position and other tools to post-process the output.

\section{Set-up for spectral library generation}

The hyperspectral library of cloud spectra is developed using this model. The library consists of spectra of nine basic cloud types. In order to generate the spectra, clouds are classified into low, medium and high opacity, each for three different levels. The number of spectra generated is 1224, 6630 and 6086 for low, medium and highly opaque clouds respectively. Thus, a total of 41,820 spectra are generated. In addition to cloud microphysical properties, several other parameters are fed into the model. They include atmospheric profiles, the underlying surface, sunsensor geometry, etc. In this case, the profiles of tropical atmosphere and a surface of mixed species are taken into account. The outputs are obtained at the height at which the aircraft is positioned.

\section{Inversion scheme}

There are different techniques for hyperspectral data matching like spectral angle mapper (SAM), spectral feature fitting (SFF), matched filtering, etc. SAM is one of 
the most widely prevalent hyperspectral classification algorithms which is based on the measure of similarity between the reference spectrum and image pixel spectrum. Since we do not have actual spectra for clouds and we rely on simulated spectra, SAM is a good option since it is insensitive to illumination, using only vector direction and not vector length. The similarity is determined by calculating the angle between the curves, where the spectra are treated as vectors in a space with dimensionality equal to the number of bands ${ }^{20}$.

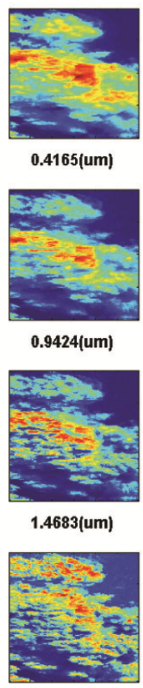

1.9942 (um)
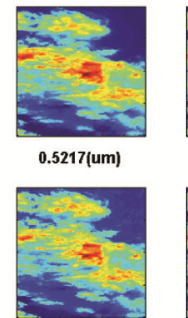

1.0476 (um)

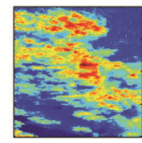

1.5735 (um)

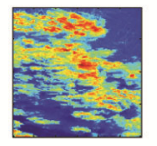

2.0994(um)

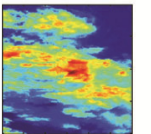

0.6269 (um)

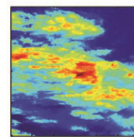

0.7321 (um)

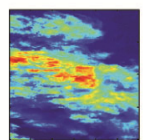

$1.1528(u m)$

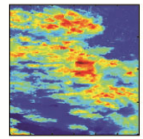

1.6787 (um)

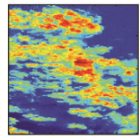

2.2046 (um)

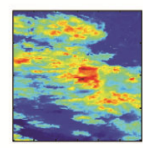

1.258 (um)

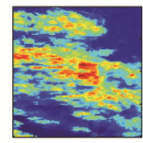

1.7839 (um)

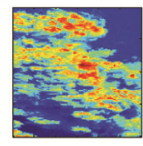

2.3098(um)

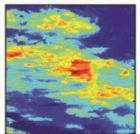

0.8372 (um)

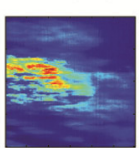

1.3631 (um)

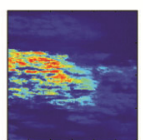

1.8891 (um)

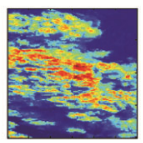

2.415(um)
Figure 3. Cloud image scene through different spectral channels of AVIRIS-NG, showing signature of cloud properties at different wavelengths.

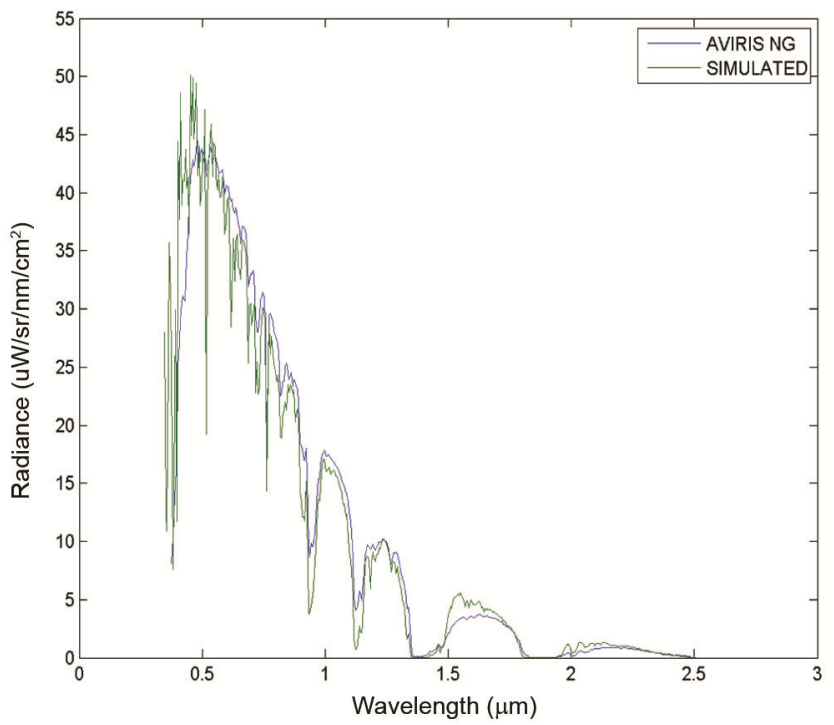

Figure 4. Plot representing the spectral signatures of observed AVIRIS-NG (blue) and simulated (green) spectra. This is one of the best matches obtained for low clouds with medium opacity.

\section{Results and discussion}

Channel selection is an important aspect for the characterization of cloud microphysical properties. Figure 3 shows the schematic of a cloudy image scene (Kurnool) visualized through different AVIRIS-NG channels. Cloud images in VIS and SWIR channels are used to explore the variability in each band. Cloud features are more distinguishable near the 1.67 and $2.2 \mu \mathrm{m}$ bands. This characteristic of SWIR bands is used in the derivation of CMPs.

The SAM technique has been applied to those scenes which are identified to be cloudy, and for which, cloud properties are mapped. The observations have been carried out during winter monsoon period which is less sampled, in contrast to summer monsoon period where sufficient data are sampled. Figure 4 shows an instance

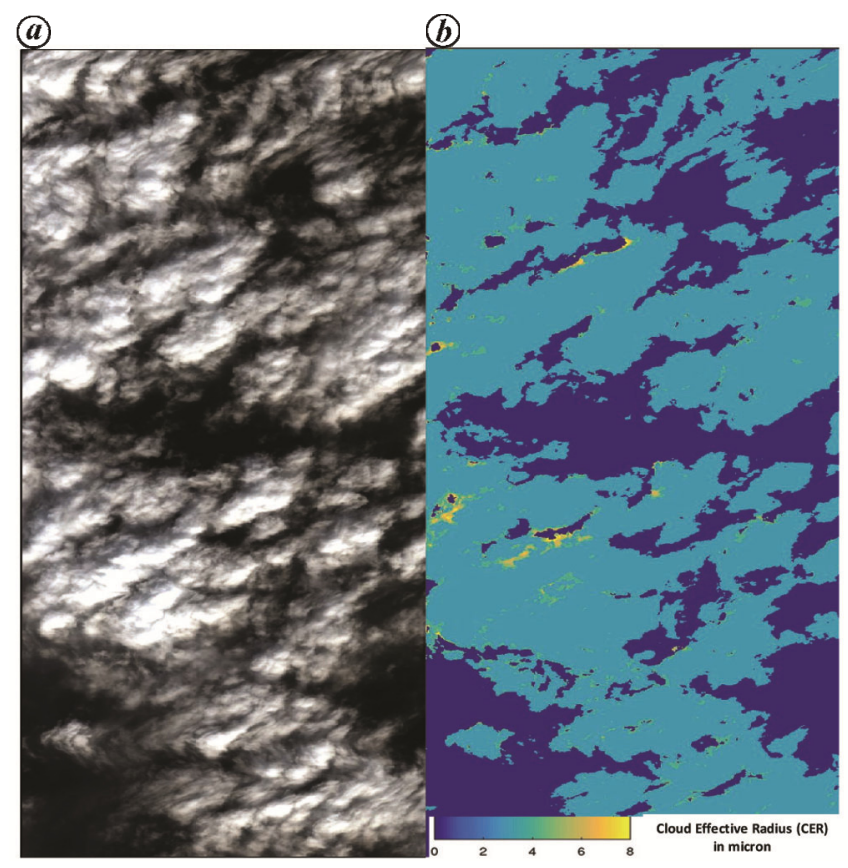

Figure 5. (a) Cloud (RGB) image and (b) retrieved microphysical parameter (cloud effective radius, $\mu \mathrm{m}$ ) from AVIRIS-NG at Chilika lagoon site, 25 December 2015.

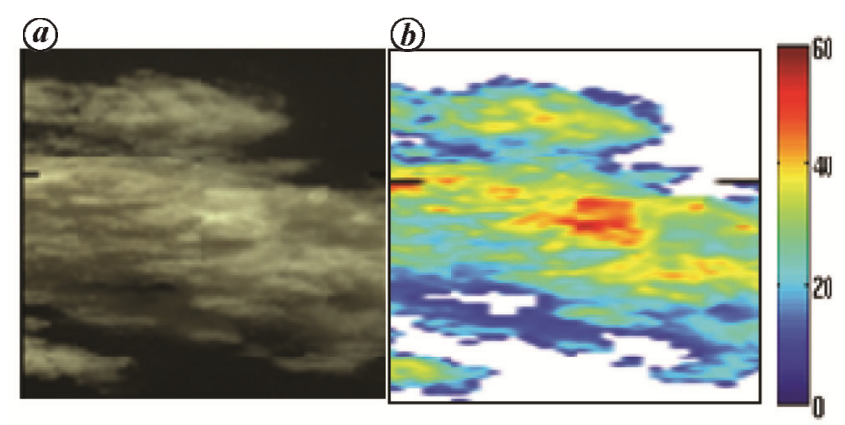

Figure 6. (a) Cloud (RGB) image and (b) retrieved microphysical parameter (cloud effective radius, $\mu \mathrm{m}$ ) from AVIRIS-NG at Kurnool site, 26 January 2016. 


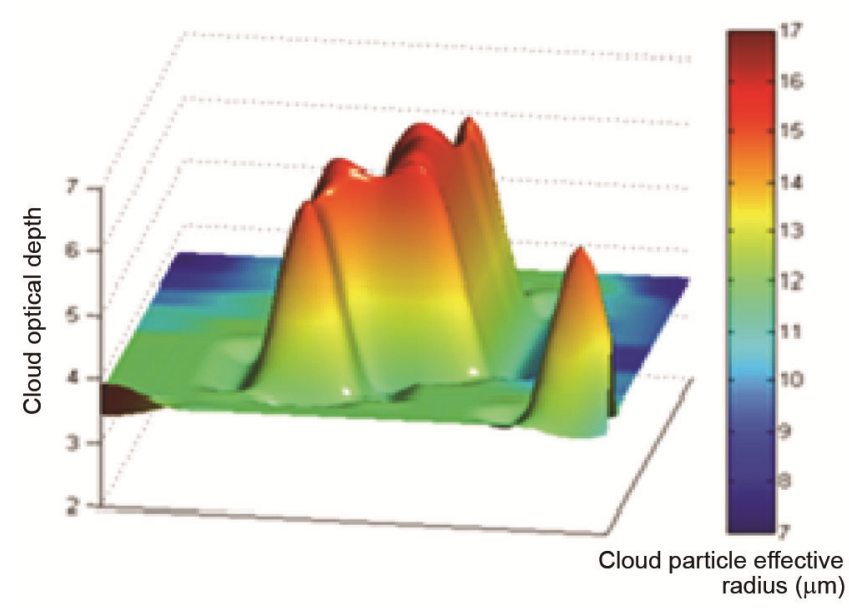

Figure 7. 3D map of cloud microphysical parameters where height represents cloud optical thickness and colors represent cloud effective radius.

where good similarity between AVIRIS-NG and simulated spectra is obtained for the same scene as Kurnool. The figure shows the spectral signature for a sample image pixel in the scene, where COT and CER are derived to be 3.6 and $4 \mu \mathrm{m}$ respectively. This is one of the best matches obtained between the RT-generated and measured spectra. The same procedure has been extended to full AVIRIS-NG scenes and corresponding CMP maps have been generated. Figures 5 and 6 present results for two sites, i.e. Chilika lagoon and Kurnool respectively. It is observed that clouds identified at Chilika region are associated with smaller droplets, which are more likely cirrocumulus clouds. The maximum CER values derived are found to be approximately $8 \mu \mathrm{m}$. However, majority of the pixels are dominated with lower CER values, viz. 2-4 $\mu \mathrm{m}$. Further, COT values are found to be very low and are in the range 3-6. This corroborates the in situ observations at Gadanki, Andhra Pradesh during winter monsoon season.

In the case of Kurnool region, one can observe spectra of image pixels associated with larger particles. Cloudy pixels with CER reaching $20 \mu \mathrm{m}$ are found in this case, with most of values above $5 \mu \mathrm{m}$. The derived parameters indicate that the clouds are relatively denser with larger particle size, which may be associated with growing cumulus clouds. The dissimilarities in CER values may be attributed to the different cloud types, e.g. cirrocumulus and growing cumulus clouds observed at both the sites. Figure 7 depicts a 3D cloud map generated for Kurnool site using COT and CER. It shows clouds of medium opacity which are associated with CER above $11 \mu \mathrm{m}$.

From the figure, we can observe that there are regions which have higher CER $(14-17 \mu \mathrm{m})$ and also relatively higher COT. A zone of high COT along with high CER implies dense clouds of bigger particles, which may lead to high precipitation rates. These $3 \mathrm{D}$ maps are possible due to very high resolution spatial and hyperspectral measurements and are useful to pick fast-developing cloud cells.

\section{Conclusion}

The present study utilizes hyperspectral measurements from AVIRIS-NG to derive CMPs. It has been observed that the channels from 1.57 to $1.78 \mu \mathrm{m}$ and 2.09 to $2.30 \mu \mathrm{m}$ are more effective in bringing out features within the clouds, revealing CER distributions in the scene. Comparing the libRadtran-simulated radiances with the observed spectra, we were able to derive the CMPs (COT and CER) from the best matches, which have been represented in the form of a 3D map. The CER distributions from the Chilika lagoon site and Kurnool site show differences in their range, which might be a result of the different atmospheric conditions at the respective sites.

1. Goetz, A. F., Vane, G., Solomon, J. E. and Rock, B. N., Imaging spectrometry for earth remote sensing. Science, 1985, 228(4704), 1147-1153.

2. Ben-Dor, E., Schläpfer, D., Plaza, A. J. and Malthus, T., Hyperspectral remote sensing. In Airborne Measurements for Environmental Research: Methods and Instruments (eds Wendisch, M. and Brenguier, J. L.), 2013, pp. 413-456.

3. Heiden, U., Segl, K., Roessner, S. and Kaufmann, H., Determination of robust spectral features for identification of urban surface materials in hyperspectral remote sensing data. Remote Sensing Environ., 2007, 111(4), 537-552.

4. Hörig, B., Kühn, F., Oschütz, F. and Lehmann, F., HyMap hyperspectral remote sensing to detect hydrocarbons. Int. J. Remote Sensing, 2001, 22(8), 1413-1422.

5. Govender, M., Chetty, K. and Bulcock, H., A review of hyperspectral remote sensing and its application in vegetation and water resource studies. Water $S A, 2007, \mathbf{3 3}(2), 145-151$.

6. Adam, E., Mutanga, O. and Rugege, D., Multispectral and hyperspectral remote sensing for identification and mapping of wetland vegetation: a review. Wetlands Ecol. Manage., 2010, 18(3), 281296.

7. Rotstayn, L. D., Indirect forcing by anthropogenic aerosols: a global climate model calculation of the effective-radius and cloud-lifetime effects. J. Geophys. Res.: Atmos., 1999, 104(D8), 9369-9380.

8. Rosenfeld, D., Woodley, W. L., Lerner, A., Kelman, G. and Lindsey, D. T., Satellite detection of severe convective storms by their retrieved vertical profiles of cloud particle effective radius and thermodynamic phase. J. Geophys. Res.: Atmos., 2008, 113(D4), D04208.

9. Harikishan, G., Padmakumari, B., Maheskumar, R. S., Pandithurai, G. and Min, Q. L., Macrophysical and microphysical properties of monsoon clouds over a rain shadow region in India from ground-based radiometric measurements. J. Geophys. Res. Atmos., 2014, 119; doi:10.1002/2013JD020872; Hirsh, E., Agassi, E. and Koren, I., Determination of optical and microphysical properties of thin warm clouds using ground based hyper-spectral analysis. Atmos. Meas. Tech., 2012, 5, 851-871.

10. King, N. J. and Vaughan, G., Using passive remote sensing to retrieve the vertical variation of cloud droplet size in marine stratocumulus: an assessment of information content and the potential for improved retrievals from hyperspectral measurements. J. Geophys. Res.: Atmos., 2012, 117(D15), 1-15. 
11. Green, R. O. et al., Imaging spectroscopy and the airborne visible/infrared imaging spectrometer (AVIRIS). Remote Sensing Environ., 1998, 65(3), 227-248.

12. Green, R. O., Bhattacharya, B. K., Eastwood, M. L., Saxena, M., Thompson, D. R. and Sadasivarao, B., Overview of the joint NASA-ISRO imaging spectroscopy science campaign in India. In AGU Fall Meeting Abstracts, 2016.

13. Hamlin, L., Green, R. O., Mouroulis, P., Eastwood, M., Wilson, D., Dudik, M. and Paine, C., Imaging spectrometer science measurements for terrestrial ecology: AVIRIS and new developments. In IEEE Aerospace Conference, Big Sky, Montana, USA, 2011, pp. 1-7.

14. Thorpe, A. K. et al., Mapping methane concentrations from a controlled release experiment using the next generation airborne visible/infrared imaging spectrometer (AVIRIS-NG). Remote Sensing Environ., 2016, 179, 104-115.

15. Bue, B. D. et al., Real-time atmospheric correction of AVIRIS-NG Imagery. IEEE Trans. Geosci. Remote Sensing, 2015, 53(12), 6419-6428.

16. Clark, R. N., Swayze, G. A., Wise, R., Livo, K. E., Hoefen, T., Kokaly, R. F. and Sutley, S. J., USGS digital spectral library splib06a. US Geological Survey, Digital Data Series, 2007, 2007, 231.

17. Baldridge, A. M., Hook, S. J., Grove, C. I. and Rivera, G., The ASTER spectral library version 2.0. Remote Sensing Environ., 2009, 113(4), 711-715.
18. Mayer, B. and Kylling, A., The libRadtran software package for radiative transfer calculations - description and examples of use. Atmos. Chem. Phys., 2005, 5(7), 1855-1877.

19. Kruse, F. A., Lefkoff, A. B., Boardman, J. W., Heidebrecht, K. B., Shapiro, A. T., Barloon, P. J. and Goetz, A. F. H., The spectral image processing system (SIPS) - interactive visualization and analysis of imaging spectrometer data. Remote Sensing Environ., 1993, 44(2-3), 145-163.

20. Jayeshlal, G. S. et al., Lidar studies on the optical characteristics of high altitude cirrus clouds at a low latitude station, Gadanki $\left(13.5^{\circ} \mathrm{N}, 79.2^{\circ} \mathrm{E}\right)$ India. Int. Arch. Photogramm., Remote Sensing Spatial Inf. Sci., 2014, 40(8), 253.

ACKNOWLEDGEMENTS. We thank the Director, SAC (ISRO), Ahmedabad for his keen interest in this work. We also thank Dr Rajkumar, Dr C. M. Kishtawal and Dr R. M. Gairola (SAC, ISRO) for their valuable suggestions during the course of this study; Dr Bimal K. Bhattacharya, Science team leader AVIRIS-NG (SAC, ISRO) for continuous guidance and support. We acknowledge the efforts of the entire AVIRIS-NG science team and thank the anonymous reviewers for their valuable suggestions that helped improve this manuscript.

doi: $10.18520 / \mathrm{cs} / \mathrm{v} 116 / \mathrm{i} 7 / 1196-1200$ 